\title{
Effect of Thermal Annealing Temperatures on MSM Photo Detector Based on Ge-like Micro Flowers in the Dark Mode
}

\author{
M.J. Jawad, Haider Y. Hammod, Amer B. Dheyab* \\ Ministry of science and technology/Directorate of material Research /center of laser research \\ *Corresponding author, e-mail: amer.aljoburi@yahoo.com'
}

\begin{abstract}
In this research, a current density of $\mathrm{J}=7.5 \mathrm{~mA} / \mathrm{cm} 2$ was applied over a constant duration of $2 \mathrm{~h}$ to grow Ge-like microflowers on Si. To fabricate a photodetector, Pd was deposited by RF-sputtering as metal contact with Ge. The samples were subjected to rapid thermal annealing (RTA) in ambient N2 at $100,200^{\circ}$ and $300^{\circ} \mathrm{C}$ for $10 \mathrm{~min}$. By screening the samples from electrical noise, the electrical characteristics through current-voltage measurements were carried out at room temperature before and after annealing. The measurements were performed in the dark, white light and UV illuminations. The forward I-V characteristics were analyzed using standard thermionic emission relation for electron transport from Metal-semiconductor-metal(MSM)with low doping concentration. The saturation current was obtained as the intercept from the straight line of In I versus V, T. It was found that at $5 \mathrm{~V}$, these currents were $2.45 \times 10-5,5.77 \times 10-5,6.12 \times 10-4$ A, respectively. Also characteristics of the MSM photodetector, linear and logarithmic forward and reverse bias, at different annealing temperatures: as-deposited, $100^{\circ} \mathrm{C}$, $200^{\circ} \mathrm{C}$ and $300^{\circ} \mathrm{C}$ in the dark mode.
\end{abstract}

Keywords: photodetector, light and UV illuminations, Ge-like microflowers, effect of thermal annealing

Copyright $\odot 2015$ Institute of Advanced Engineering and Science. All rights reserved.

\section{Introduction}

A metal-semiconductor-metal photodetector (MSM detector) is a photo-detector device containing two Schottky contacts, i.e., two metallic electrodes on a semiconductor material, in contrast to a $p-n$ junction as in a photodiode. During operation, some electric voltage is applied to the electrodes. When light impinges on the semiconductor between the electrodes, it generates electric carriers (electrons and holes), which are collected by the electric field and thus can form a photocurrent. MSM detectors can be made faster than photodiodes. Their detection bandwidths can reach hundreds of gigahertz, making them suitable for very highspeed optical fiber communications. Consist of interdigitated metallic electrode deposited on top of a semiconductor material with which it forms Schottky contacts Broad band width, high sensitivity with a low capacitance and dark current, low cost of fabrication and ease of monolithic integration responsivity is low due to the shadowing effect backside illuminated structure can be used or thin semi transparent electrodes can be grown [1, 2]. Advantage of MSM photodetector strong electric field in the active area pure drift photocurrent, no diffusion component and very fast photo response, determined by saturation velocity, vs. MSM photodetector based on Ge nanostructures Optical detectors are applied in all fields of human activities from basic research to commercial applications in communication, automotive, medical imaging, homeland security, and other fields. The fundamental purpose of any photodetector is to convert optical signals into electrical signals, usually expressed as a photocurrent, which can be amplified, displayed and/or retransmitted. Ge has been attractive as an alternative substrate to Si because Ge has higher carrier mobility over Si and good compatibility with Si processing [3]. Due to its optical properties, $\mathrm{Ge}$ is a good candidate for the development of light detectors operating in the near infrared region (NIR: 800-1600 nm) especially when integration with Sibased devices are required. Homojunction Ge photodiodes can be fabricated on top of graded [4] and thin constant composition. SixGe1-x/Si buffer layers [5]. Alternatively, Ge can be deposited directly on Si without any intermediate SiGe layer. Si can then act both as a substrate and as one of the diode terminals. Examples are $p-i-n$ heterojunction photodiodes based on 
crystalline [6], poly crystalline and amorphous $\mathrm{Ge}$ [7]. In the last two decades there has been a stable research interest in the development of high-speed photodetectors in the form of rectifying metalsemiconductor-metal photodetector (MSM-PD). Based on an interdigitated Schottky barrier structure, the MSMPD has a simple device technology, low dark current, fast response, small capacitance and large active area. Most of the beneficial properties of the MSM-PD stem from its lateral planar geometry. The contacts of the MSM-PD are from two interdigitated, forksshaped that function as back-to-back Schottky diodes. The basic structure of the MSM-PD is illustrated in Figure 1.

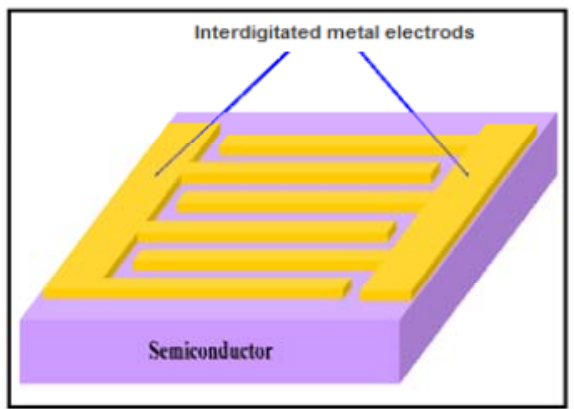

Figure 1. A schematic image of an MSM photodiode structure

One of the advantages of the interdigitated MSM detector is that finger width and spacing as well as the light sensitive area can easily be adjusted with a simple mask change. Some experimental and theoretical results demonstrating response time dependence on the MSM-PD geometry has already been reported in the past [8, 9].

\section{Theory}

The device works by absorbing optical energy and converting a photon flux into a time varying electrical signal. When the active areas of the device are under illumination, carriers in the semiconductor absorption layer (also known as electronhole pairs) are generated by incident photons having energy greater than the bandgap energy. The carriers are transported to the metal contact pads, and a current is detected in the external circuit under the application of an external bias voltage.When a voltage is externally applied on two electrodes system, it biases them so that one is in the forward and the other is in the reverse direction. A comparison of the energy band diagram of Schottky and MSM photodetector is illustrated in Figure 2.
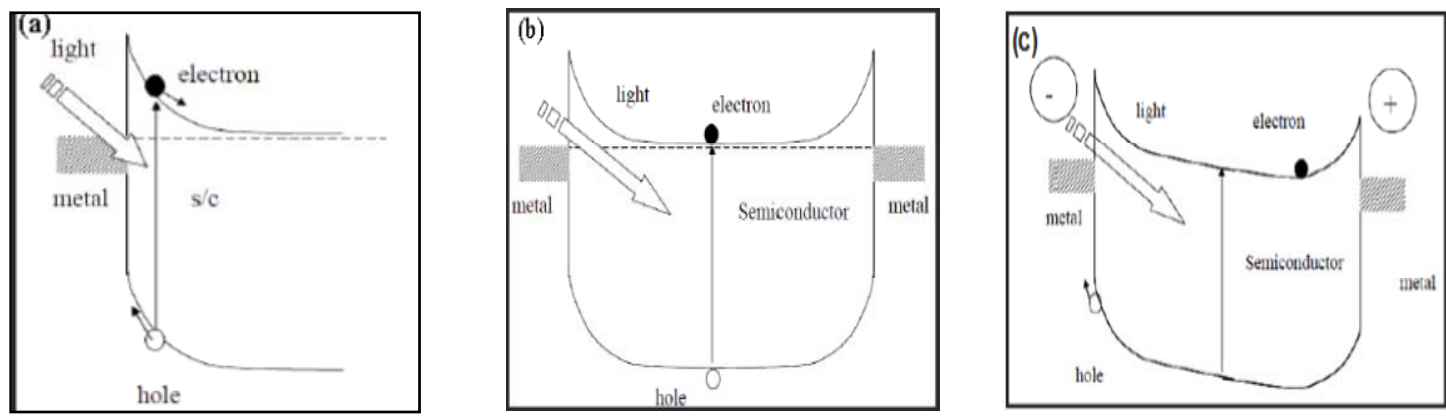

Figure 2. Energy band diagram of (a) Schottky photodetector and (b) MSM photodetector at zero bias, and (c) MSM photodetector at moderate bias

When the bias voltage is zero, Schottky photodiode produces a certain amount of photo current and the electric field is zero at the center since the structure of the MSM is symmetrical. Photo- 
excited electrons are trapped in the potential well and the net photocurrent is zero (Figure 2(b)). When the bias is moderate, the potential barrier at the forward contact is decreased as shown in Figure 2(c). There is an electric field between the Schottky electrodes. The majority of photoelectrons are trapped in the potential well and the photo-holes are not, but cannot leave the active region because of the charge of the trapped electrons. The net photocurrent is very small. When the bias is raised, the potential barrier for photoelectrons disappears and the depletion region will be widened and eventually becomes equal to electrode separation. The carrier transport mechanism is similar as in Schottky photodiode by the transport of photogenerated electrons from the semiconductor to the metal over the potential barrier and the carriers injection from the metal into the semiconductor which contribute to the dark current. A photon with energy greater than the bandgap will be absorbed by an electron exciting it to the conduction band. The most important features of any photodetector are represented by the responsivity, quantum efficiency, response time series resistance, contrast ratio and current gain. To study the effective Schottky barrier height SBH, the famous model that can be used to determine current-voltage (I-V) characteristics is the thermionic emission theory, According to this theory, the current (Id) from the semiconductor to the metal can be expressed as:

$$
I_{d}=I_{o} \exp \left(\frac{q V_{d}}{n k T}\right)\left[1-\exp \left(\frac{-q V_{d}}{k T}\right)\right]
$$

Where $\mathrm{n}$ is the ideality factor which may depend on temperature and independent on voltage, $\mathrm{Vd}$ is the voltage across the diode, $\mathrm{k}$ is the Boltzman constant, and lo is the saturation current given by:

$$
I_{o}=A A^{* *} T^{2} \exp \left[\frac{-q \varphi_{b}}{k T}\right]
$$

Where $q$ is the electron charge, $T$ the temperature, $A$ is the contact area, $A^{* *}$ is the effective Richardson constant and $\left(\varphi_{b}\right)$ is the Schottky barrier height. Equation (1) at $V>3 \mathrm{kT} / \mathrm{q}$, become:

$$
I_{d}=I_{o} \exp \left(\frac{q V_{d}}{n k T}\right)
$$

The theoretical value of $\mathrm{A}^{* *}$ can be calculated using $\mathrm{A}^{* *}=4 \pi \mathrm{m}^{*} \mathrm{qk} 2 / \mathrm{h} 3$ and based on the effective mass $m^{*}$. The plot of In I vs $V$ will give a straight line with a slope of $q /(n k T)$, and the intercept with $y$-axis will yield lo, in which barrier height, $\varphi b$ can be obtained by using Equation (2) and the ideality factor from the slope. A top view of SEM image of PS sample prepared over 30 min etching time is shown in Figure 4(a). For this sample, which is prepared at the optimized condition, a uniform distribution of pores with size of $100 \sim 200 \mathrm{~nm}$ was identified. A side view of the porous network cross section indicates that the dimensional length is ranged from 85 to $95 \mu \mathrm{m}$ as illustrated in Figure 3(b). The growth of Ge film was obtained by a simple and low-cost ECD technique, where this method yielded a large area of Ge microstructures. Figure 3(c) and (d) represent respectively, low and high magni-fication SEM images for the entangled Ge microrods in low dimensions that formed on the Si. The lengths of the microrods were about several microns and had an average diameter of about 500 200 nm. Figure 3(e) illustrates a typical SEM image in low magnification of Ge microspheres with nanowires net grown on PS substrate. The distribution extends to cover the whole surface of $\mathrm{Ge}$ microspheres with a diameter of about $500 \mathrm{~nm}$. Figure 3(f) shows a typical high-magnification SEM image with more details of the net formed under the Ge microspheres. This net has dimensions of less than $100 \mathrm{~nm}$ in diameter and a length of about few microns. The EDX on the top right of Figure 3(c) and Figure 3(e) confirmed the growth of $\mathrm{Ge}$ on both substrates. The results showed that the amount of $\mathrm{O}$ was found to be very low relative to the existing $\mathrm{Ge}$. 


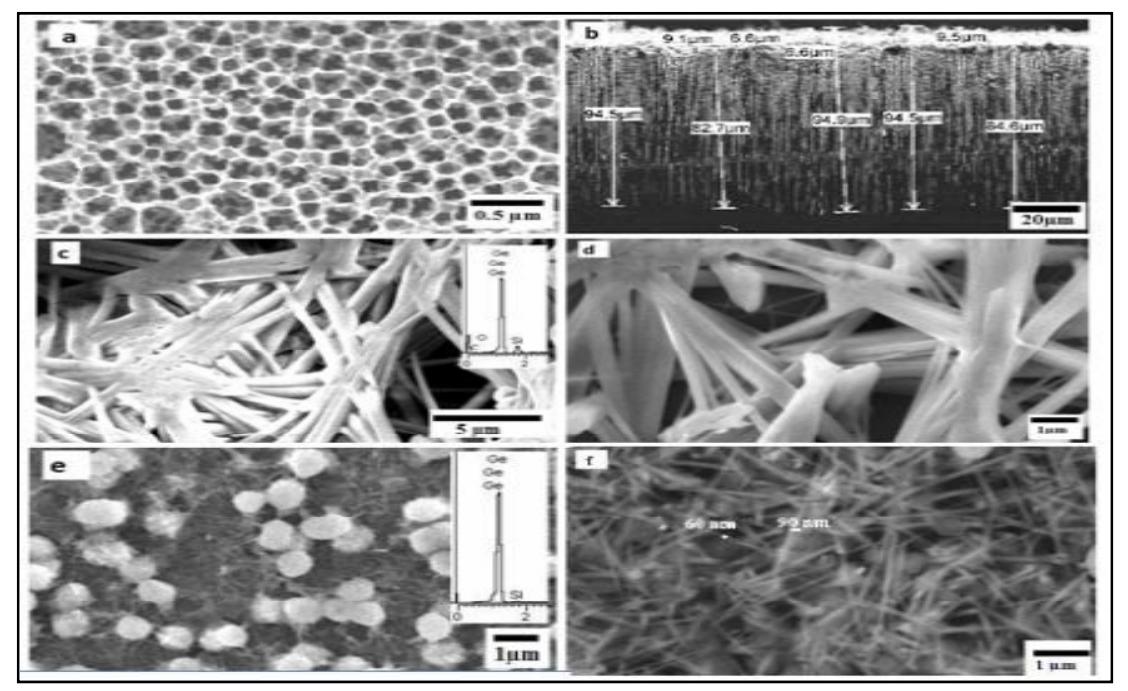

Figure 3. SEM images (a) surface of PS sample, (b) cross section of PS sample, (c) low and (d) high magnification SEM images of $\mathrm{Ge}$ microrods on $\mathrm{Si}$, (e) microspheres with nanowires in low magnification and (f) high magnification of Ge nanowires on PS

The Ge microspheres were closely attached with the PS substrate as shown in the SEM image of Figure 3(e). This could be attributed to the partial filling of the Ge particles in the pores, and thus lead to improve the structural stability of the porous silicon substrate. Hence, the PS surface had a significant effect on the size and shape of the Ge nanowires.

\section{Discuss the Results}

The Schottky barrier heights and ideality factor were calculated from Equation (2) and (3). In addition, the series resistance, contrast ratio, and the current gain. All these calculations were carried out for the as-grown and annealed MSM photodetectors in white light and UV illumination and the results are listed in Table 1.

Table 1. Barrier height, ideality factor, series resistance, contrast ratio and current gain for as grown and annealed MSM photodetectors measured under white light and UV illuminations.

\begin{tabular}{ccccccc}
\hline $\begin{array}{c}\text { Temp. } \\
\left({ }^{\circ} \mathrm{C}\right)\end{array}$ & Photodetectors & $\begin{array}{c}\text { Barrier } \\
\text { height } \\
\varphi \mathrm{b}(\mathrm{eV})\end{array}$ & $\begin{array}{c}\text { Ideality } \\
\text { factor } \mathrm{n}\end{array}$ & $\begin{array}{c}\text { Series } \\
\text { resistance } \\
\mathrm{R}(\mathrm{k} \Omega)\end{array}$ & $\begin{array}{c}\text { Contrast } \\
\text { Ratio at 5V }\end{array}$ & $\begin{array}{c}\text { Current } \\
\text { gain }\end{array}$ \\
\hline \multirow{2}{*}{ As grown } & Light & 0.725 & 1.46 & 81.37 & 2.35 & 10 \\
& UV & 0.672 & 1.1 & 8.34 & 24.9 & 50 \\
100 & Light & 0.707 & 1.31 & 40.7 & 11.75 & 20 \\
& UV & 0.662 & 1.0 & 5.56 & 93.5 & 75 \\
200 & Light & 0.684 & 1.19 & 16.3 & 47 & 50 \\
& UV & 0.636 & 0.9 & 2.08 & 398.8 & 200 \\
300 & Light & 0.693 & 1.2 & 23.3 & 41.15 & 35 \\
& UV & 0.644 & 1.03 & 2.78 & 373.8 & 150 \\
\hline
\end{tabular}

Annealed samples exhibited more significant change compared to the asdeposited MSM photodetector. It was observed that the barrier height and the ideality factor for the detector decreased with increasing temperature until $200^{\circ} \mathrm{C}$ then started to rise again. The barrier height decreases from $0.725 \mathrm{eV}$ to $0.684 \mathrm{eV}$ with the incident white light and from 0.672 to $0.636 \mathrm{eV}$ with UV light when the temperature is elevated from 25 to $200^{\circ} \mathrm{C}$, accompanied by a noticeable improvement of the ideality factor $n$ which decreases from 1.46 to 1.19 and 1.1 to 0.9 , respectively. The contrast ratio, represented by the ratio of photocurrent to the dark current at $5 \mathrm{~V}$, which was calculated for annealed samples and found to increase with increasing 
temperature with values of 47 and 398 at $200^{\circ} \mathrm{C}$ for white light and UV illuminations, respectively. The maximum current gains of the devices increased at annealing temperatures up to $200^{\circ} \mathrm{C}$ in both white light and UV illuminations. This could be due to the reduction of the series resistance as a function of annealing temperature up to $200^{\circ} \mathrm{C}$. Such a result contributes to low dark current from the Schottky barrier MSM photodetectors.

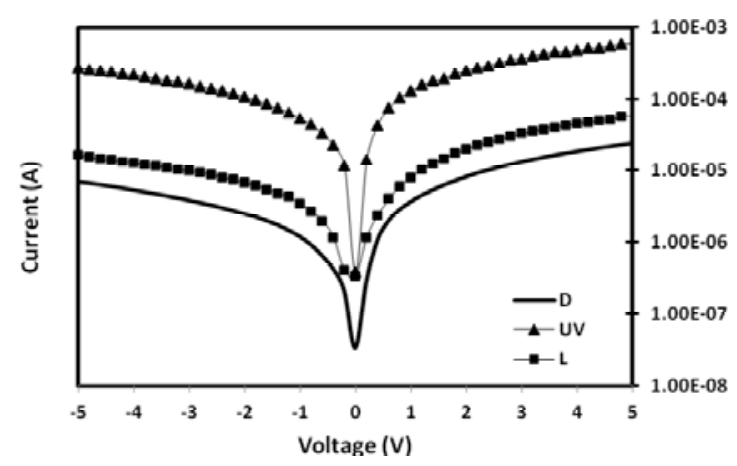

Figure 4. Characteristics of the as grown MSM photodetector based on Ge like microflowers under dark, white light and UV illuminations

From Figure 4, the response of the Pd MSM photodetector increases with the bias voltage and it saturates gradually when the voltage is beyond $2 \mathrm{~V}$. The current starting at a low voltage is typical of thermionic emission current. In the linear region, the thermionic emission and the carrier velocity increase with the biasing voltage. The dark current and the photocurrent are saturated because all carriers are swept out towards the contacts. It can be noticed that the MSM photodetector produces lower dark current than that of those of white light and UV illuminations at $5 \mathrm{~V}$ bias. This shows that the light illumination can effectively create electron-hole pairs in $\mathrm{Ge}$ and greatly increase the carrier density. Under illumination, light impinges onto the Ge layer, the high energy photons are absorbed by this layer producing more electron-hole pairs, the application of a bias voltage to the $\mathrm{Pd}$ contact creates an electric field within the underlying $\mathrm{Ge}$ layer that sweeps the photo generated carriers out of the depletion region, resulting in an increase of the photocurrent with voltage.

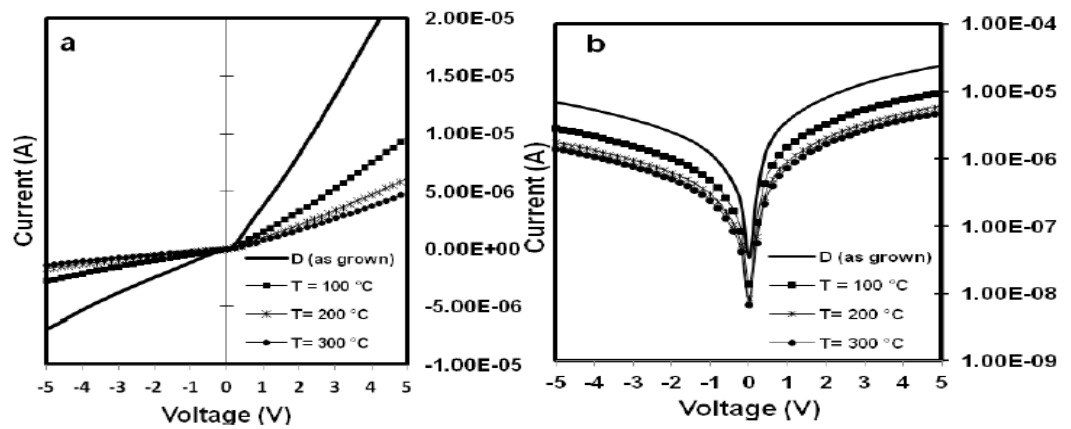

Figure 5. Characteristics of the MSM photodetector, (a) linear and (b) logarithmic forward and reverse bias, at different annealing temperatures: as-deposited, $100^{\circ} \mathrm{C}, 200^{\circ} \mathrm{C}$ and $300^{\circ} \mathrm{C}$ in the dark mode

Figures 5(a) and (b) show linear and logarithmic forward and reverse bias I-V characteristics of Pd MSM photodetector annealed at 100, 200 and $300^{\circ} \mathrm{C}$ together with that for the as-deposited diode, where all measurements were carried out in the dark mode. The dark current exhibited remarkable dependence on the annealing temperature. The dark current,

TELKOMNIKA Vol. 16, No. 3, December 2015 : $502-508$ 
which is one of the main sources of noise in photodetectors, decreases with increasing annealing temperature. The results also indicated optimum I-V characteristics established by the lowest reverse leakage current were achieved in the sample annealed at $300^{\circ} \mathrm{C}$. The variation of the Schottky barrier height with annealing temperature for Pd Schottky contacts with $\mathrm{Ge}$ is demonstrated in Figure 5. The SBH for as-grown and annealed samples for the MSM photodetector were found to vary from 0.779 to $0.820 \mathrm{eV}$ according to the alteration of the annealing temperature.

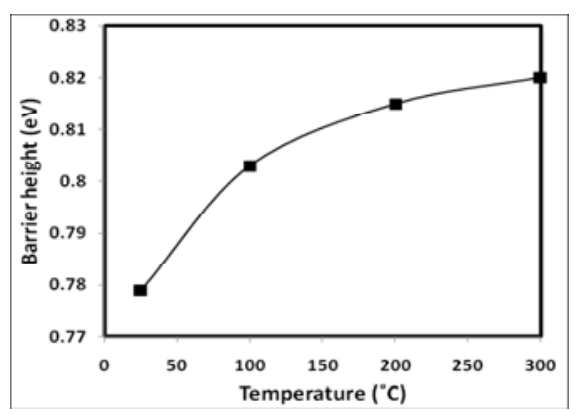

Figure 6. The dependence of Schottky barrier height on annealing temperature for the MSM photodetector

It was found that under dark condition measurements, the Schottky contact barrier height of the MSM photodetector increases with increasing annealing temperature within the range between $25-300^{\circ} \mathrm{C}$ due to the lower dark currents exhibited by the thermally-treated samples compared to that of the as deposited sample. With such observations under dark conditions, the electrical characteristics of the photodetector is said to be enhanced by the high temperature which changes $\mathrm{Ge}$ nanocrystallites layer beneath the contact to single/poly crystalline and promoting good electrical conduction. Nevertheless, previous studies have shown the variation of barrier height is nearly constant between $100-500^{\circ} \mathrm{C}$. An increment in the $\mathrm{Pd} / \mathrm{Ge}$ Schottky contacts ideality factor with increasing annealing temperature within the range between $25-300^{\circ} \mathrm{C}$ can be seen in Figure 7 . Such behavior is in good agreement with previous work published elsewhere.

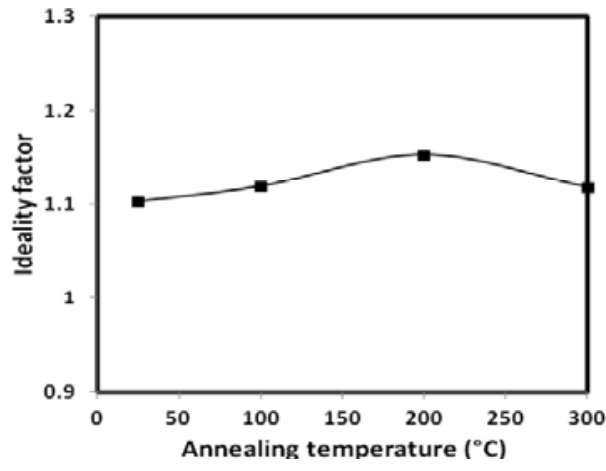

Figure 7. A graphical representation of the ideality factor as a function of annealing temperature for the MSM photodetector

\section{Conclusion}

The photoelectric properties of Ge-like microflowers grown on $\mathrm{Si}$ were studied at different annealing temperatures of 100,200 and $300^{\circ} \mathrm{C}$ in dark, white and UV illumination. The annealed samples exhibited more significant changes compared to the as-grown one. It was also observed that the barrier height and the ideality factor of the detector decreased with 
increasing temperature until $117,200^{\circ} \mathrm{C}$. The responsivity displayed two peaks, the maximum one at $400 \mathrm{~nm}$ and the second lower peak at $500 \mathrm{~nm}$. The rise time of the photodetector was $130 \mathrm{~ms}$ for $460 \mathrm{~nm}$ and $100 \mathrm{~ms}$ for $405 \mathrm{~nm}$ and the quantum efficiency was $13.61 \%$ for $400 \mathrm{~nm}$ at $5 \mathrm{~V}$.

\section{References}

[1] WC Koscielniak, et al. Dynamic behavior of photocarriers in a GaAs metal-semiconductor- metal photodetector with sub-half-micron electrode pattern. Appl. Phys. Lett. 1989; 54(6): 567.

[2] JBD Soole, H Schumacher. InGaAs metal-semiconductor-metal photodetectors for long wavelength optical communications. IEEE J. Quantum Electron. 1991; 27(3): 737.

[3] Park K, An CH, Lee MS, Yang CW, Lee HJ, Kim H. Microstructural evolution and electrical characteristics of Co-germanide contacts on Ge. J. Electrochem. Soc. 2009; 156: 229-232.

[4] Samavedam SB, Currie MT, Langdo TA, Fitzgerald EA. Highquality germanium photodiodes integrated on silicon substrates using optimized relaxed graded buffers. Appl. Phys. Lett. 1998; 73 : 2125-2127.

[5] Oehme M, Werner J, Jutzi M, Wöhl G, Kasper E, Berroth M. High bandwidth Ge p-i-n photodetector integrated on Si. Appl. Phys. Lett. 2006; 89: 71117-71119.

[6] Colace L, Balbi M, Masini G, Assanto G, Luan HC, Kimerling LC. Ge on Si p-i-n photodiodes operating at 10 Gbits. Appl. Phys. Lett. 2006; 88; 101111-101113.

[7] Masini G, Colace L, Galluzzi F, Assanto G. Advances in the field of poly-Ge on Si near infrared photodetectors. Mater. Sci. Eng. B. 2000; 257(69): 257-260.

[8] Ito M, Wada O. Low dark current GaAs metal-semiconductor-metal (MSM) photodiodes using WSixcontacts. IEEE J. Quant. Electron. 1968; 22: 1073-1077.

[9] Soole JBD, Schumacher H. InGaAs metal-semiconductor-metal photodetectors for long wavelength optical communications. IEEE J.Qunt.Electron. 1991; 27: 737-752. 